\title{
Constraint Representation and Reduction based on Lie Algebra in Assembly Model
} Hitoshi Tokunaga, Kenichiro Itakura, Fumiki Tanaka and Takeshi Kishinami
Graduate School of Engineering, Hokkaido University

\begin{abstract}
In assembly modelling, it is necessary to capture the relative positions of components in the assembly. Many such problems concern constraint representation and its manipulation such as constraint reduction. This paper formulates the constraints as groups of rigid body transformations and proposes a constraint reduction procedure, based on Lie algebra. Then, this approach is applied to the constraint representation and reduction in an assembly model.
\end{abstract}

Key words: Assembly Model, Constraint Representation, Lie Group, Lie Algebra, Constraint Reduction

\section{INTRODUCTION}

In extending solid modeling technology to the modeling of assemblies, it is important to capture the relative position of each component to other components. Many such problems concern the representation and manipulation of constraints on the relative positions of components.

A number of research papers have described various types of constraints and their manipulation, such as constraint reduction, in the assembly model ${ }^{1 \text { - }}$ 5). As shown in Figure 1(a), in the assembly model, assembly is represented by its components and their connections which are represented as contact relations between the features of the components. The relative position of the components are constrained by the constraint derived from the contact relation. As shown in Figure 1(b), the relative position of the (rigid body) components is described by a transformation matrix corresponding to the displacement between the coordinate systems of the components. The constraint is defined as the subgroup of the group $S E(3)$ (Special Euclidean 
group) formed by all the transformation matrices. However, it is not so clear how many types of constraints should be considered. Constraint reduction is the procedure that computes a single net constraint from multiple constraints, according to the operation determined by the configuration $(c f l, c f 2)$ of the constraints (See Section 5 about the configurations of constraints in detail). However, since the reduction procedures for this constraint representation need the complete table of reduction rules or complicated symbolic matrix operations, it is not easy to compute the subgroup of transformation matrices which represents the reduced constraint.

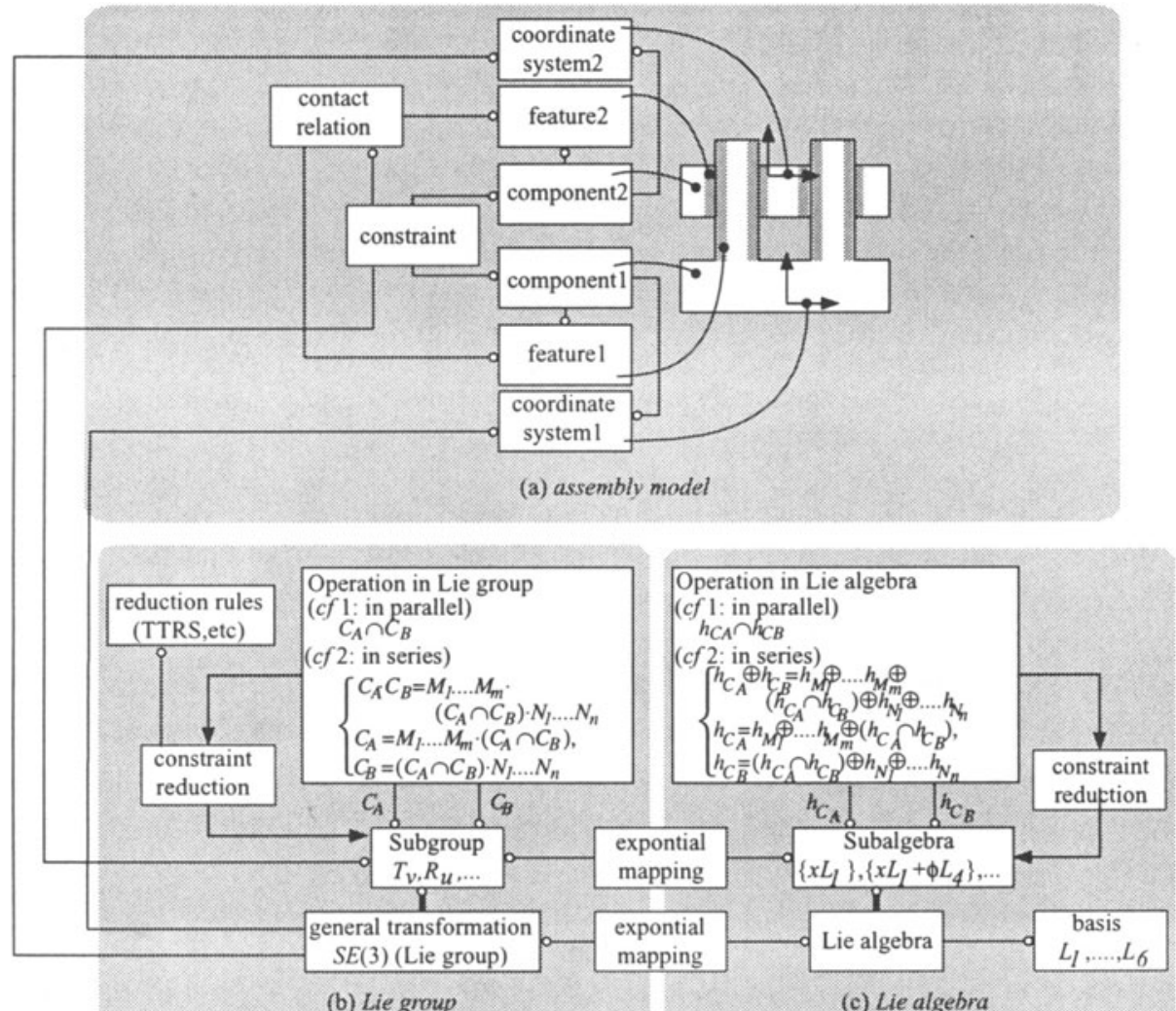

Figure 1. An assembly model, constrain representation and constraint reduction.

In this report, in order to solve these problems, the constraint representation and reduction in the assembly model is made clear theoretically as follows:

(1) Constraints are defined as the subgroups of $S E(3)$ based on Lie algebra. 
It is well-known that $S E(3)$ is a Lie group, and that the Lie group has a one-to-one correspondence to a linear vector space, called Lie algebra, by exponential mapping as shown in Figure $1(\mathrm{c})^{6)-8)}$. The Lie algebra of $S E(3)$ has a basis of six and it is easy to derive all the subalgebra because only finite combinations of the basis are checked. The subgroup of the Lie group also has a one-to-one correspondence to the subalgebra of the Lie algebra. Therefore, by deriving the Lie subalgebra, the corresponding Lie subgroup can be derived.

(2) Constraint reduction procedure is proposed based on Lie algebra as shown in Figure 1(c). In addition to the correspondence described above, the operations of the constraint reduction for the Lie group also correspond to that of Lie algebra. By using Lie algebra, the constraint reduction can be represented as a problem of solving linear equations. In this report, as the configuration of the constraints, two types of configurations are defined. The first one $(c f l)$, called constraints in parallel, is the configuration of two constraints which are specified between two rigid bodies. The other ( $c f 2)$, called constraints in series, is the configuration of two constraints which are specified between rigid bodies via another rigid body.

(3) This approach is applied to the constraint representation and reduction in an assembly model.

\section{RIGID BODY TRANSFORMATION}

The relative position of a rigid body to another can be described by a homogeneous transformation matrix corresponding to the displacement from the coordinate system of the latter body to that of the former body. The set of all such matrices forms a group $S E(3)$, the special Euclidean group of rigid body transformations, which can be represented by the following equation:

$$
S E(3)=\left\{\operatorname{Trans}(x, y, z) R_{x}(\phi) R_{y}(\theta) R_{z}(\psi) \mid x, y, z, \phi, \theta, \psi \in \mathbf{R}\right\}
$$

where $\operatorname{Trans}(x, y, z)$ represents a translation by a vector $\left(\begin{array}{ll}x & y \\ z\end{array}\right)^{t}, R_{x}(\phi), R_{y}(\theta)$, and $R_{z}(\psi)$ represent rotations about the Cartesian axes $x, y$, and $z$, which are defined as the following equation: 


$$
\begin{aligned}
& \operatorname{Trans}(x, y, z)=\left(\begin{array}{llll}
1 & 0 & 0 & x \\
0 & 1 & 0 & y \\
0 & 0 & 1 & z \\
0 & 0 & 0 & 1
\end{array}\right), R_{x}(\phi)=\left(\begin{array}{cccc}
1 & 0 & 0 & 0 \\
0 & \cos \phi & -\sin \phi & 0 \\
0 & \sin \phi & \cos \phi & 0 \\
0 & 0 & 0 & 1
\end{array}\right) \\
& R_{y}(\theta)=\left(\begin{array}{cccc}
\cos \theta & 0 & \sin \theta & 0 \\
0 & 1 & 0 & 0 \\
-\sin \theta & 0 & \cos \theta & 0 \\
0 & 0 & 0 & 1
\end{array}\right) R_{z}(\psi)=\left(\begin{array}{cccc}
\cos \psi & -\sin \psi & 0 & 0 \\
\sin \psi & \cos \psi & 0 & 0 \\
0 & 0 & 1 & 0 \\
0 & 0 & 0 & 1
\end{array}\right)
\end{aligned}
$$

In this report, the constraint is represented by the subset of $S E(3)$ which means the displacements permitted by the constraint.

\section{LIE GROUP AND LIE ALGEBRA}

$G L(n, C)$ denotes the group of regular complex matrices with matrix multiplication. It is well-known that a group $G$ is a linear Lie group if $G$ is a closed subgroup of $G L(n, C)$. Since it is easy to show that $S E(3)$ is a closed subgroup of $G L(n, C), S E(3)$ is a linear Lie group.

Assume that $G$ is a linear Lie group. The linear Lie algebra $g$ of $G$ is defined as the following equation:

$$
g=\{X \in g l(n, C) \mid \exp (t X) \in G, t \in \mathbf{R}\}
$$

where $g l(n, C)$ is the set of all the square complex matrices. The Lie algebra $g$ of a Lie group $G$ is the tangent space to $G$ at the identity, whose dimension is the same as $G$.

The exponential map is defined as follows:

$$
\exp X=\sum_{k=0}^{\infty} \frac{X^{k}}{k !}
$$

In Lie algebra $g$, a product $[X, Y](X, Y \in g)$, called the Lie bracket, is defined as follows:

$$
[X, Y]=X Y-Y X
$$


Let $s e(3)$ denote the Lie algebra of SE(3). Any element $X$ of $s e(3)$ can be expressed as follows:

$$
X=\sum_{i=1}^{6} \alpha_{i} L_{i}
$$

where $\alpha \in \mathbf{R}, L_{1}, \cdots L_{6}$ are the standard basis for $\operatorname{se}(3)$, which are represented as follows:

$$
\begin{aligned}
& L_{1}=\left(\begin{array}{llll}
0 & 0 & 0 & 1 \\
0 & 0 & 0 & 0 \\
0 & 0 & 0 & 0 \\
0 & 0 & 0 & 0
\end{array}\right), L_{2}=\left(\begin{array}{llll}
0 & 0 & 0 & 0 \\
0 & 0 & 0 & 1 \\
0 & 0 & 0 & 0 \\
0 & 0 & 0 & 0
\end{array}\right), L_{3}=\left(\begin{array}{llll}
0 & 0 & 0 & 0 \\
0 & 0 & 0 & 0 \\
0 & 0 & 0 & 1 \\
0 & 0 & 0 & 0
\end{array}\right) \\
& L_{4}=\left(\begin{array}{cccc}
0 & 0 & 0 & 0 \\
0 & 0 & -1 & 0 \\
0 & 1 & 0 & 0 \\
0 & 0 & 0 & 0
\end{array}\right) L_{5}=\left(\begin{array}{cccc}
0 & 0 & 1 & 0 \\
0 & 0 & 0 & 0 \\
-1 & 0 & 0 & 0 \\
0 & 0 & 0 & 0
\end{array}\right), L_{6}=\left(\begin{array}{cccc}
0 & -1 & 0 & 0 \\
1 & 0 & 0 & 0 \\
0 & 0 & 0 & 0 \\
0 & 0 & 0 & 0
\end{array}\right)
\end{aligned}
$$

$L_{1}, \ldots, L_{6}$ satisfy the following equations:

$$
\begin{aligned}
& \exp \left(x L_{1}\right)=\operatorname{Trans}(x, 0,0), \exp \left(y L_{2}\right)=\operatorname{Trans}(0, y, 0), \exp \left(z L_{3}\right)=\operatorname{Trans}(0,0, z), \\
& \exp \left(\phi L_{4}\right)=R_{x}(\phi), \exp \left(\theta L_{5}\right)=R_{y}(\theta), \exp \left(\psi L_{6}\right)=R_{z}(\psi)
\end{aligned}
$$

Table 1 shows the bracket products of all the combinations of $L_{1}, \ldots, L_{6}$.

Table 1. Bracket product $[A, B]$ of $L_{l}, \ldots L_{6}$.

\begin{tabular}{c|c|c|c|c|c|c}
\hline A & $L_{1}$ & $L_{2}$ & $L_{3}$ & $L_{4}$ & $L_{5}$ & $L_{6}$ \\
\hline$L_{1}$ & 0 & 0 & 0 & 0 & $L_{3}$ & $-L_{2}$ \\
\hline$L_{2}$ & 0 & 0 & 0 & $-L_{3}$ & 0 & $L_{1}$ \\
\hline$L_{3}$ & 0 & 0 & 0 & $L_{2}$ & $-L_{1}$ & 0 \\
\hline$L_{4}$ & 0 & $L_{3}$ & $-L_{2}$ & 0 & $L_{6}$ & $-L_{5}$ \\
\hline$L_{5}$ & $-L_{3}$ & 0 & $L_{1}$ & $-L_{6}$ & 0 & $L_{4}$ \\
\hline$L_{6}$ & $L_{2}$ & $-L_{1}$ & 0 & $L_{5}$ & $-L_{4}$ & 0 \\
\hline
\end{tabular}




\section{CONSTRAINT REPRESENTATION}

The subset $h$ of the Lie algebra $g$ is a subalgebra of $g$ if $h$ is closed under the bracket product, that is, if $[X, Y] \in h$ whenever $X$ and $Y$ belong to $h$. Since all the elements of the subset are represented by the linear combination of the basis, the bracket product between the elements can be represented as follows:

$$
[X, Y]=\left[\sum_{i=1}^{N_{X}} \alpha_{i} L_{i}^{X}, \sum_{j=1}^{N_{Y}} \beta_{j} L_{j}^{Y}\right]=\sum_{i=1}^{N_{X}} \sum_{j=1}^{N_{Y}} \alpha_{i} \beta_{j}\left[L_{i}^{X}, L_{j}^{Y}\right]
$$

where $\alpha_{i}, \beta_{j} \in \mathbf{R}, L_{i}^{X}$ and $L_{j}^{Y}$ are the basis, $N_{X}$ and $N_{Y}$ are the numbers of the basis. Therefore, by checking the bracket product of the basis, it is possible to check whether $h$ is a subalgebra or not. When a Lie group $G$ and a Lie algebra $g$ of $G$ are given, it is well-known that there is a one-to-one correspondence between a Lie subalgebra $h$ of $g$ and a Lie connected subgroup $H$ of $G$. Therefore, by deriving all the Lie subalgebra from $s e(3)$, all the subgroups of $S E(3)$ can be derived. In this report, the constraints are defined by using these subgroups. For example, let's consider the subset determined by $L_{1}$ and $L_{4}$. From Table 1 , it is clear that this subset is closed under the bracket product, and that this subset forms a subalgebra of $s e(3)$. The corresponding subgroup of $S E(3)$ can be described as follows:

$$
\begin{aligned}
C & =\left\{\exp \left(x L_{1}+\phi L_{4}\right) \mid x, \phi \in \mathbf{R}\right\}=\left\{\exp \left(x L_{1}\right) \exp \left(\phi L_{4}\right) \mid x, \phi \in \mathbf{R}\right\} \\
& =\left\{\operatorname{Trans}(x, 0,0) R_{x}(\phi) \mid x, \phi \in \mathbf{R}\right\}
\end{aligned}
$$

The constraint represented by this subgroup is denoted as $C_{u}$. In the same way, ten types of constraints are derived as shown in Table 2.

Table 2. Derived constraints.

\begin{tabular}{c|c|l|l|l}
\hline DOF & Notation & Elements of subgroup & Basis of Lie algebra & Lower pair \\
\hline \multirow{3}{*}{1} & $T_{v}$ & $\operatorname{Trans}(x, 0,0)$ & $L_{1}$ & Prismatic \\
& $R_{u}$ & $R_{x}(\phi)$ & $L_{4}$ & Revolute \\
& $H_{u, p}$ & $\operatorname{Trans}(x, 0,0) R_{x}(p x)$ & $L_{1}+p L_{4}(p:$ constant $)$ & Helical \\
\hline \multirow{2}{*}{2} & $T_{p}$ & $\operatorname{Trans}(0, y, z)$ & $L_{2}, L_{3}$ & \\
& $C_{u}$ & $\operatorname{Trans}(x, 0,0) R_{x}(\phi)$ & $L_{1}, L_{4}$ & Cylindrical \\
\hline \multirow{4}{*}{3} & $T$ & $\operatorname{Trans}(x, y, z)$ & $L_{1}, L_{2}, L_{3}$ & \\
& $G_{p}$ & $\operatorname{Trans}(0, y, z) R_{x}(\phi)$ & $L_{2}, L_{3}, L_{4}$ & Planar \\
& $S_{o}$ & $R_{x}(\phi) R_{y}(\theta) R_{z}(\psi)$ & $L_{4}, L_{5}, L_{6}$ & Spherical \\
& $Y_{u, p}$ & $\operatorname{Trans}(x, y, z) R_{x}(p x)$ & $L_{1}+p L_{4}, L_{2}, L_{3}$ & \\
\hline 4 & $X_{v}$ & $\operatorname{Trans}(x, y, z) R_{x}(\phi)$ & $L_{1}, L_{2}, L_{3}, L_{4}$ & \\
\hline
\end{tabular}




\section{CONSTRAINT REDUCTION}

If the relative positions of two rigid bodies are constrained by two or more constraints, the net effect may be reduced to a single constraint. Such a procedure is called constraint reduction. Two types of configurations of constraints are defined in this report. The first one, called constraints in parallel, is the configuration in the case of two contact relations $\left(C R_{A}\right.$ and $\left.C R_{B}\right)$ which are specified between the features of two rigid bodies $\left(A_{l}\right.$ and $A_{2}$ ) as shown in Figure 2(a). The other, called constraints in series, is the configuration in the case of one contact relation $\left(C R_{A}\right)$ which is specified between the features of two rigid bodies $\left(A_{1}\right.$ and $\left.A_{2}\right)$ and the other contact relation $\left(C R_{B}\right)$ is specified between the feature of one of these rigid bodies $\left(A_{2}\right)$ and the feature of another body $\left(A_{3}\right)$ as shown in Figure 2(b).

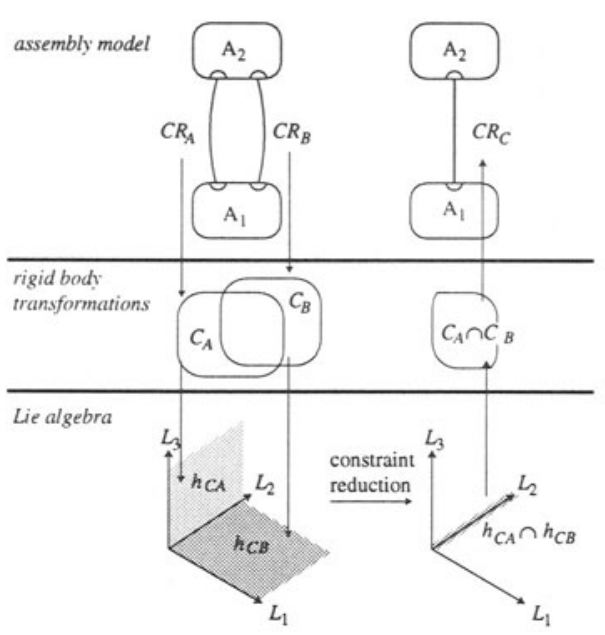

(a) constraints in parallel

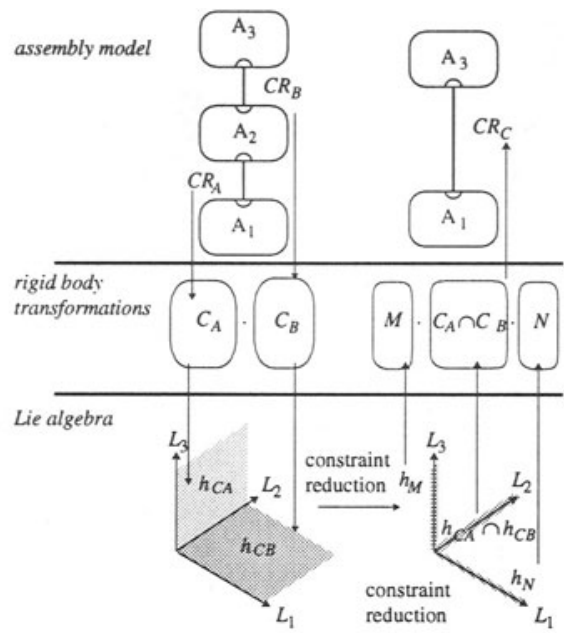

(b) constraints in series

Figure 2. Constraint reduction.

The reduction procedure for the constraints in parallel is defined as the procedure for deriving the following constraint as shown in Figure 3(a):

$$
\begin{aligned}
& D_{11} H_{A} D_{21}^{-1} \cap D_{12} H_{B} D_{22}^{-1}=\left\{D_{11} X D_{21}^{-1} \mid X \in H_{A}\right\} \cap\left\{D_{12} Y D_{22}^{-1} \mid Y \in H_{B}\right\} \\
& =\left\{D_{11} X D_{21}^{-1} \mid D_{11} X D_{21}^{-1}=D_{12} Y D_{22}^{-1}, X \in H_{A}, Y \in H_{B}\right\} \\
& =\left\{D_{11} X D_{21}^{-1} \mid X D_{21}^{-1} D_{22} D_{12}^{-1} D_{11}=D_{11}^{-1} D_{12} Y D_{12}^{-1} D_{11}, X \in H_{A}, Y \in H_{B}\right\}
\end{aligned}
$$


where $D_{i j} \in S E(3)$ is the displacement from the coordinate system $F_{A i}$ of the rigid body $A_{i}$ to the coordinate system $F_{f j A i}$ of the feature $j$ of $A_{i}$, and $H_{A}$ and $H_{B}$ are the subgroups of the displacements from $F_{f I A I}$ to $F_{f I A 2}$ and from $F_{f 2 A I}$ to $F_{f 2 A 2}$ as the representation of contact relations $C R_{A}$ and $C R_{B}$, respectively. If the specified contact relations are consistent, the following equation is satisfied:

$$
D_{21}^{-1} D_{22} D_{12}^{-1} D_{11} \in H_{A} \text {. }
$$

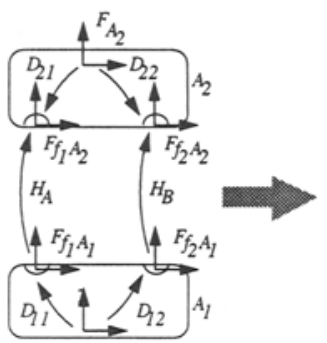

(a) $F_{A_{I}}$

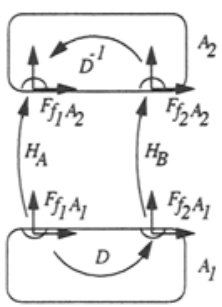

(b)

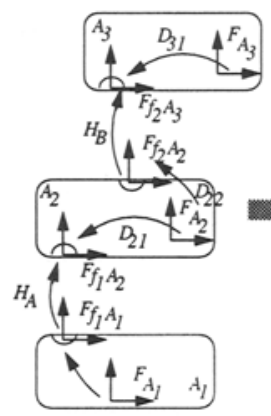

(c)

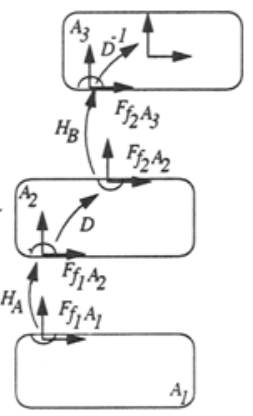

(d)

Figure 3. Constraint representation.

Therefore, Equation (11) becomes the following equation:

$$
\begin{aligned}
& \left\{D_{11} X D_{21}^{-1} \mid X=D Y D^{-1}, X \in H_{A}, Y \in H_{B}\right\} \\
& =D_{11}\left(\left\{X \mid X \in H_{A}\right\} \cap\left\{D Y D^{-1} \mid Y \in H_{B}\right\} D_{21}^{-1}\right. \\
& =D_{11}\left(H_{A} \cap D H_{B} D^{-1}\right) D_{21}^{-1}
\end{aligned}
$$

where $D=D_{11}^{-1} D_{12}$. Therefore, by computing the following equation as shown in Figure 2(a) and Figure 3(b), the reduced constraint represented by Equation (13) can be obtained.

$$
C_{A} \cap C_{B}
$$

where $C_{A}=H_{A}, C_{B}=D H_{B} D^{-1}$.

It is known that the product set of Lie subgroups corresponds to the product set of the corresponding Lie subalgebra. Thereby, in Lie algebra, the constraint reduction procedure can be defined as the procedure for deriving the following subalgebra as shown in Figure 2(a): 


$$
h_{C A} \cap h_{C B}
$$

where Lie algebra (or subalgebra) of constraint $C$ is denoted as $h_{C}$. By representing the Lie subalgebra $\left(h_{C A}\right.$ and $\left.h_{C B}\right)$ with the basis $\left(L_{i}^{A}\right.$ and $\left.L_{i}^{B}\right)$ of Lie algebra shown in Table 2, Equation (15) is represented by the following equation:

$$
\begin{aligned}
& \left\{A \mid A=\sum_{i=1}^{N_{A}} \alpha_{i} L_{i}^{A}, \alpha_{i} \in \mathbf{R}\right\} \cap\left\{B \mid B=\sum_{i=1}^{N_{B}} \beta_{i} D L_{i}^{B} D^{-1}, \beta_{i} \in \mathbf{R}\right\} \\
& =\left\{A \mid A=\sum_{i=1}^{N_{A}} \alpha_{i} L_{i}^{A}=\sum_{i=1}^{N_{B}} \beta_{i} D L_{i}^{B} D^{-1}, \alpha_{i}, \beta_{i} \in \mathbf{R}\right\}
\end{aligned}
$$

where $N_{A}$ and $N_{B}$ are the numbers of basis. That is, the reduced constraint can be obtained by deriving $\alpha_{i}$ and $\beta_{i}$ that satisfy the following equation:

$$
\sum_{i=1}^{N_{A}} \alpha_{i} L_{i}^{A}=\sum_{i=1}^{N_{B}} \beta_{i} D L_{i}^{B} D^{-1}
$$

Next, before showing the other reduction procedure, the composition of constraints $\left(C_{i}\right.$ and $\left.C_{j}\right)$ is defined as follows:

$$
C_{i} \cdot C_{j}=\left\{X \cdot Y \mid X \in C_{i}, Y \in C_{j}\right\} .
$$

The reduction procedure for the constraints in series is defined as the procedure for deriving the following constraint as shown in Figure 3(c):

$$
\begin{aligned}
D_{11} H_{A} D_{21}^{-1} \cdot D_{22} H_{B} D_{31}^{-1} & =\left\{D_{11} X D_{21}^{-1} \mid X \in H_{A}\right\} \cdot\left\{D_{22} Y D_{31}^{-1} \mid Y \in H_{B}\right\} \\
& =D_{11}\left(H_{A} \cdot D H_{B} D^{-1}\right) D D_{31}^{-1}
\end{aligned}
$$

where $D=D_{21}^{-1} D_{22}, D_{i j} \in S E(3)$ is the displacement from the coordinate system $F_{A i}$ of the rigid body $A_{i}$ to the coordinate system $F_{f j A i}$ of the feature $j$ of $A_{i}$, and $H_{A}$ and $H_{B}$ are the subgroups of the displacements from $F_{f l A I}$ to $F_{f l A 2}$ and from $F_{f 2 A 2}$ to $F_{f l A 3}$ as the representation of contact relations $C R_{A}$ and $C R_{B}$, respectively. By computing the following equation as shown in Figure 2(b) and Figure 3(c), the reduced constraint represented by Equation (19) can be obtained. 


$$
C_{A} \cdot C_{B}=M_{1} \ldots M_{m} \cdot\left(C_{A} \cap C_{B}\right) \cdot N_{1} \ldots N_{n}
$$

where $C_{A}=H_{A}, C_{B}=D H_{B} D^{-1}, M_{1} \ldots M_{m}$ and $N_{l} \ldots N_{n}$ are the constraints that satisfy the following equations:

$$
\begin{aligned}
& C_{A}=M_{1} \ldots M_{m} \cdot\left(C_{A} \cap C_{B}\right), \\
& C_{B}=\left(C_{A} \cap C_{B}\right) \cdot N_{l} \ldots N_{n} .
\end{aligned}
$$

The Lie algebra $h_{M l}, \ldots, h_{M m}$ and $h_{N l}, \ldots, h_{N n}$ are represented by the basis of and $h_{C A}$ and $h_{C B}$, respectively, which are not those of $h_{C A} \cap h_{C B}$ as shown in Figure 2 (b). The Lie algebra $h_{M l}, \ldots, h_{M m}$ and $h_{N l}, \ldots, h_{N n}$ satisfy the following equations:

$$
\begin{aligned}
& h_{C A}=h_{M I} \oplus \ldots \oplus h_{M m} \oplus\left(h_{C A} \cap h_{C B}\right) \\
& h_{C B}=\left(h_{C A} \cap h_{C B}\right) \oplus h_{N I} \oplus \ldots \oplus h_{N n}
\end{aligned}
$$

where $\oplus$ means the direct sum.

\section{EXAMPLES OF CONSTRAINT REDUCTION}

When two contact relations $\left(C R_{A}\right.$ and $\left.C R_{B}\right)$ in parallel which represent cylindrical contacts are specified as shown in Figure 4(a), the elements $h_{C A}$ and $h_{C B}$ of the corresponding constraints $C_{A}$ and $C_{B}$ can be represented by $x L_{1}+\phi L_{4}$ and $D\left(x^{\prime} L_{1}+\phi^{\prime} L_{4}\right) D^{-1}$ from Table 2 , respectively. The elements of $h_{C A} \cap h_{C B}$ satisfy the following equation:

$$
x L_{1}+\phi L_{4}=D\left(x^{\prime} L_{1}+\phi^{\prime} L_{4}\right) D^{-1}
$$

If the center axes ( $x$-axes) of cylinders are parallel, $D$ can be represented by the following equation:

$$
D=\operatorname{Trans}(a, b, c) R_{x}(\alpha)
$$

where $b \neq 0$, or $c \neq 0$. By substituting $D$ into Equation (25) and deriving the basis, the following equation is obtained: 


$$
x L_{1}+\phi L_{4}=x^{\prime} L_{1}+\phi^{\prime}\left(c L_{2}-b L_{3}+L_{4}\right) .
$$

By solving it, the following solutions are derived:

$$
x=x^{\prime}, \phi=\phi^{\prime}=0 .
$$

Therefore, $C_{A} \cap C_{B}$ is obtained as follows:

$$
C_{A} \cap C_{B}=\left\{\exp \left(x L_{1}\right) \mid x \in \mathbf{R}\right\}=\{\operatorname{Trans}(x, 0,0) \mid x \in \mathbf{R}\} .
$$

This constraint allows only prismatic motion for $A_{2}$ with respect to $A_{1}$ as shown in Figure 4(b), and it is denoted by $T_{v}$.

When two contact relations $\left(C R_{A}\right.$ and $\left.C R_{B}\right)$ in series which represent cylindrical contacts are specified and the center axes ( $x$-axes) of cylinders are parallel as shown in Figure 4(c), the elements of $h_{C A}$ and $h_{C B}$ of $C_{A}$ and $C_{B}$ are represented by $x L_{1}+\phi L_{4}$ and $D\left(x L_{1}+\phi L_{4}\right) D^{-1}=x^{\prime} L_{1}+\phi^{\prime}\left(c L_{2}-b L_{3}+L_{4}\right)$ which are the same as the example above. Since the base of $h_{C A} \cap h_{C B}$ is $L_{l}$, the base of $h_{M 1}$ is $L_{4}$ and that of $h_{N 1}$ is $c L_{2}-b L_{3}+L_{4}$. Therefore, $C_{A} \cdot C_{B}$ is derived as follows:

$$
\begin{aligned}
& C_{A} \cdot C_{B}=M_{1} \cdot\left(C_{A} \cap C_{B}\right) \cdot N_{1} \\
& =\left\{\exp \left(\phi L_{4}\right) \mid \phi \in \mathbf{R}\right\} \cdot\left\{\exp \left(x L_{1}\right) \mid x \in \mathbf{R}\right\} \cdot\left\{\exp \left(\phi^{\prime}\left(c L_{2}-b L_{3}+L_{4}\right)\right) \mid \phi^{\prime} \in \mathbf{R}\right\} \\
& =\left\{\exp \left(x L_{1}\right) \exp \left(\phi L_{4}\right) \mid x, \phi \in \mathbf{R}\right\} \cdot\left\{D \exp \left(\phi^{\prime} L_{4}\right) D^{-1} \mid \phi^{\prime} \in \mathbf{R}\right\} \\
& =\left\{\operatorname{Trans}(x, 0,0) R_{x}(\phi) \mid x, \phi \in \mathbf{R}\right\} \cdot\left\{D R_{x}\left(\phi^{\prime}\right) D^{-1} \mid \phi^{\prime} \in \mathbf{R}\right\}
\end{aligned}
$$

This constraint means the composition of two constraints as shown in Figure 4(d), and it is denoted by $C_{u} \cdot R_{u}$.

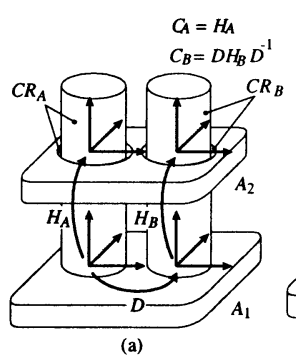

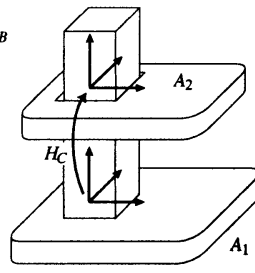

(b)



(d)

Figure 4. Examples of constraint reduction 


\section{CONCLUSIONS}

In this report, in order to make theoretically clear the constraint representation and reduction in an assembly model, the group theory was applied to them as follows:

1. Based on Lie algebra, it was made clear that there were ten types of subgroups in $S E$ (3), and the constraints were represented by using them,

2. A constraint reduction procedure was proposed based on Lie algebra. By using Lie algebra, the constraint reduction could be represented as a problem of solving linear equations.

3. This approach was applied to constraint representation and reduction in an assembly model.

\section{ACKNOWLEDGMENTS}

This research work has been financially supported by a Grant-in-Aid for JSPS Fellows and the Research Fellowships of the Japan Society for the Promotion of Science (JSPS) for Young Scientists.

\section{REFERENCES}

1) Clement, A., Desrochers, A., and Riviere, A., Theory and Practice of 3-D Tolerancing for Assembly, Proc. CIRP Int. Working Semi. Computer-Aided Tolerancing, 1991, 25-55.

2) Kim, S. H., and Lee, K., An Assembly Modelling System for Dynamic and Kinematic Analysis, Computer-Aided Design, vol. 21, no. 1, 1989, 2-12.

3) Popplestone, R. J., Liu, Y., and Weiss, R., A Group Theoretic Approach to Assembly Planning, AI Magazine, Spring, 1990, 82-97.

4) Thomas, F., and Torras, C., A Group-Theoretic Approach to the Computation of Symbolic Part Relations, IEEE Journal of Robotics and Automation, vol. 4, no. 6, 1988, 622-634.

5) Turner, J. U., Subramaniam, S., and Gupta, S., Constraint Representation and Reduction in Assembly Modeling and Analysis, IEEE Transaction on Robotics and Automation, vol. 8, no. 6, 1992, 741-750.

6) Karger, A., and Novak, J., Space Kinematics and Lie Groups, Gordon and Breach Science Publishers, New York, 1985.

7) Knapp, A. W., Lie group beyond an introduction, Birkhauser, Boston, 1996.

8) Sattinger, D. H., and Weaver, O. L., Lie Groups and Algebras with Applications to Physics, Geometry, and Mechanics, Springer-Verlag, New York, 1986. 